Prada Jiménez de Cisneros, Joaquín, Matthews, Louise, Mair, Colette, Stefan, Thorsten, and Stear, Michael J (2014) The transfer of IgA from mucus to plasma and the implications for diagnosis and control of nematode infections. Parasitology, 141 (7). pp. 875-879. ISSN 0031-1820

Copyright (c) 2014 Cambridge University Press

A copy can be downloaded for personal non-commercial research or study, without prior permission or charge

Content must not be changed in any way or reproduced in any format or medium without the formal permission of the copyright holder(s)

When referring to this work, full bibliographic details must be given

http://eprints.gla.ac.uk/94631/

Deposited on: 23 June 2014

Enlighten - Research publications by members of the University of Glasgow http://eprints.gla.ac.uk 


\title{
The transfer of IgA from mucus to plasma and the implications for diagnosis and control of nematode infections
}

\author{
JOAQUÍN PRADA JIMÉNEZ DE CISNEROS*, LOUISE MATTHEWS, COLETTE MAIR, \\ THORSTEN STEFAN and MICHAEL J. STEAR
}

\author{
Institute of Biodiversity, Animal Health and Comparative Medicine, University of Glasgow, Garscube Campus, \\ Bearsden Road, Glasgow G61 1QH, UK
}

(Received 29 August 2013; revised 16 December 2013; accepted 17 December 2013; first published online 13 February 2014)

\begin{abstract}
SUMMARY
Immunoglobulin A ( $\operatorname{IgA}$ ) activity has been associated with reduced growth and fecundity of Teladorsagia circumcincta. $\operatorname{IgA}$ is active at the site of infection in the abomasal mucus. However, while IgA activity in abomasal mucus is not easily measured in live animals without invasive methods, IgA activity can be readily detected in the plasma, making it a potentially valuable tool in diagnosis and control. We used a Bayesian statistical analysis to quantify the relationship between mucosal and plasma IgA in sheep deliberately infected with T. circumcincta. The transfer of IgA depends on mucosal IgA activity as well as its interaction with worm number and size; together these account for over $80 \%$ of the variation in plasma IgA activity. By quantifying the impact of mucosal IgA and worm number and size on plasma IgA, we provide a tool that can allow more meaningful interpretation of plasma IgA measurements and aid the development of efficient control programmes.
\end{abstract}

Key words: Modelling, Teladorsagia circumcinta, parasite, immunoglobulin A, sheep, immunology.

\section{INTRODUCTION}

In sheep, IgA activity against fourth-stage larvae is strongly and consistently associated with reduced worm growth and fecundity following infection with the abomasal parasite Teladorsagia circumcincta (Stear et al. 1995; Balic et al. 2003; MartinezValladares et al. 2005). The site of infection is the abomasum, but collecting abomasal tissue or mucus requires invasive surgery or post-mortem sampling. Therefore plasma IgA has been widely used to study the build-up of immunity over the grazing season and the sources of variation among animals (Strain et al. 2002; Henderson and Stear, 2006). The relationship between mucosal and plasma $\operatorname{IgA}$ is consequently of critical importance for the interpretation of $\operatorname{Ig} \mathrm{A}$ activity. In particular, the construction of immunologically explicit models of the host-parasite system is only possible if this relationship is understood. The relationship between mucosal and plasma IgA is influenced by IgA activity in the abomasal mucus and the number of nematodes in the abomasum (Sinski et al. 1995; Stear et al. 1995). Mucosal IgA will bind to nematodes and excess IgA will move into the lymph and subsequently appear in the plasma.

However, adult $T$. circumcincta differ in size. Some sheep have populations of relatively small

* Corresponding author: Institute of Biodiversity, Animal Health and Comparative Medicine, University of Glasgow, Garscube Campus, Bearsden Road, Glasgow G61 1QH, UK. E-mail: Joaquin.Prada@glasgow.ac.uk worms while other sheep harbour much larger worms (Stear et al. 1998). Therefore we investigated whether the size of the adult nematodes also influenced the relationship between mucosal and plasma $\operatorname{IgA}$. We have produced a three-dimensional surface plot of the relationship between mucosal $\operatorname{IgA}$, worm mass (worm length $\times$ worm number) and plasma $\operatorname{IgA}$. In addition, we have used this relationship to predict the likely distribution of mucosal IgA.

MATERIALS AND METHODS

\section{Experimental data}

The experimental data used in the statistical analysis and to infer the distributions of the parameters are from two published studies. One study monitored the response to deliberate infection in Scottish Blackface sheep (Stear et al. 1995). This study measured mucosal and plasma IgA activity against fourthstage larvae, worm burdens and mean worm lengths. These data were used to quantify the relationship between mucosal and plasma IgA. The second study was based on five successive cohorts, each of 200 lambs from a naturally infected commercial flock in southwest Strathclyde (Stear et al. 1998), which were monitored for plasma IgA, worm burden and worm length. This second study was used to explore the possible distribution of mucosal IgA.

The deliberate infection study was conducted with 30 six-month-old female lambs chosen randomly from a flock of 200 Scottish Blackface lambs which 
had been exposed to natural, mixed, predominantly $T$. circumcincta infection. They were transferred to the Veterinary School and housed together indoors under conditions to minimize extraneous infection. After 3 months, 24 of the 30 lambs were infected with 50000 infective third-stage $T$. circumcincta larvae. After 8 weeks, all sheep were treated with two broadspectrum anthelmintics and 4 weeks later reinfected with 50000 larvae. Three sheep were infected only at the second date and served as infection controls, while the other 3 were not infected and served as uninfected controls. All sheep were monitored for another 8 weeks and necropsied.

Mean worm length was calculated by measuring the length of 100 female adult worms from the abomasal contents of each animal. Only one worm was recovered from one lamb, which was dropped from the analysis. Worm number was calculated in both experiments by counting the worms in a $2 \%$ sample of abomasum wash from each animal and multiplying by 50 (Strain, 2001). The abomasal mucosa from each animal was digested and a $2 \%$ sample was used to estimate the number of fourthstage larvae (Armour et al. 1966).

Indirect ELISA with biotinylated monoclonal antibodies was used to measure mucosal and plasma IgA activity. The cell line used to generate monoclonal IgG anti-sheep IgA (M1521) were kindly donated by Dr S. Hobbes, Dr P. Bird and Professor I. McConnell (personal communication) (Strain, 2001). Larval antigens were prepared from somatic and excretory-secretory material as described by Sinski et al. (1995).

\section{Statistical analysis}

The relationship between plasma IgA, mucosal IgA and worm biomass was explored in a Bayesian framework. A similar relationship between plasma $\operatorname{IgA}$, mucosal $\operatorname{IgA}$ and worm burden, rather than worm biomass, has previously been explored using a general linear model by Stear et al. (1995). As there is no informative prior information, a Bayesian analysis is expected to give similar results to a classical frequentist analysis. However, a Bayesian framework provides a more natural method for future work in this area.

For the purposes of this analysis, the worm mass in individual sheep was estimated as mean adult female worm length multiplied by the number of adult worms. This approximates worm mass, but the actual worm mass would require a knowledge of worm diameter, deviation from a cylindrical shape, the length of adult males and the variation in worm length among individual worms. To some extent, the coefficients in the regression relationship allow for the deviation from true worm mass. A logarithmic transformation was used to normalize the

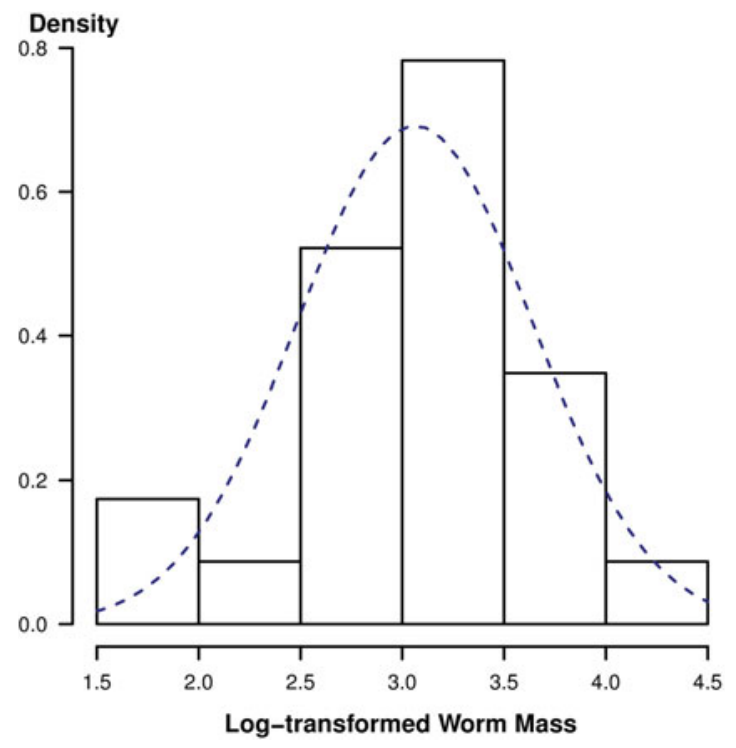

Fig. 1. Distribution of the $\log$ transformed estimated total worm mass in individual sheep $(n=23)$ overlaid with the best-fitting normal distribution estimated by maximum likelihood $($ mean $=3 \cdot 06$; s.D. $=0 \cdot 58)$.

distribution of the worm mass. The log-transformed distribution of worm mass in the 23 sheep was not significantly different from a normal distribution (Fig. 1; Shapiro-Wilk test, $P=0 \cdot 21$ ), although with only 23 animals it is not a very powerful test of the distribution.

The intercept and the main effect of worm mass were found not significant after fitting the full model (intercept, the two main effects and the interaction of mucosal IgA and worm mass), so these variables were dropped from the equation. The final model equation, hereafter referred to as the IgA transfer equation, therefore only included the effects of mucosal $\operatorname{IgA}$ and the interaction between mucosal IgA and worm mass.

$I g A_{p}=\alpha_{1} \times I g A_{m}+\alpha_{2} \times I g A_{m} \times \log _{10}(W M)+\varepsilon$

This model was fitted using a Bayesian approach, which returns the distribution of possible values (called the posterior distribution) for each coefficient; if zero lies inside the $95 \%$ credible interval, that coefficient is viewed as non-significant (Gelman et al. 2004). $\alpha_{1}$ and $\alpha_{2}$ are the regression coefficients being calculated while $\varepsilon$ is the error term, deviation of the obtained value from the true $\operatorname{Ig} A_{p}$ value, which accounts for the variation that is not explained by the model.

The DIC (deviance information criterion) was used to compare different models (Spiegelhalter et al. 2002), with the preferred model being the one with the lowest DIC. We also calculated a penalized $R^{2}$ which estimates the proportion of the total variation in plasma $\operatorname{IgA}$ activity that was explained by the variation in mucosal IgA and worm mass, while 
accounting for changes in the number of variables being fitted (Faraway, 2006).

The statistical distribution of mucosal IgA levels among individual sheep is not known. We therefore tested whether a normal distribution of mucosal IgA was compatible with the known distributions of plasma IgA and the number of $T$. circumcincta in the host. Plasma $\operatorname{IgA}$ follows a gamma distribution (Murphy et al. 2010), the number of T. circumcincta follows a negative binomial (Stear et al. 1998) while worm length is normally distributed (Stear et al. 1995). We revised the model equation to infer mucosal IgA, hereafter referred to as reverse transfer equation, from the plasma IgA and worm mass (calculated again as the product of mean adult female length and number of adult worms) data in the natural infection study (Stear et al. 1998). We then explored potential distributions of mucosal IgA. This dataset has a larger number of animals; therefore tests to explore the distribution are more powerful. Extreme predicted IgA values were discarded because they may have arisen from errors in the estimation of worm number; we used the ShapiroWilk procedure to test departures from normality and the Kolmogorov-Smirnov test to compare the fitted gamma distribution to the mucosal IgA distribution among lambs.

Statistical analyses were carried out using the R language (R Development Core Team, 2011) and JAGS (a Bayesian Analysis program using Gibbs sampling; Plummer, 2003). The Bayesian regression analysis was run using uninformative priors, two chains were used with 500000 iterations and a thinning of 40 ; the burn-in period was 10000 . The model was run twice, first as a full model with all the parameters and then a simplified model with only the statistically significant parameters from the previous fitting. Type III sums of squares (SS) were calculated to obtain the $R^{2}$; unlike commonly used type I SS, type III SS do not depend on the order in which effects are specified in the model.

Figures were plotted using the $\mathrm{R}$ language (R Development Core Team, 2011). To draw the surface plot, a grid of mucosal IgA activity and worm mass values was created (from 0 to 2 and 0 to 10000 respectively) with 50 intermediate nodes on each axis. The plasma IgA values were calculated for each point of the grid from the transfer equation and the surface was then interpolated from these points. Worm mass origin was changed to 200 (the first node) for graphic convenience to avoid artefacts being created at very low worm biomass.

\section{RESULTS}

Using the Bayesian model, plasma IgA activity was dependent on mucosal $\operatorname{IgA}$ activity and the interaction between worm mass and mucosal IgA activity (Table 1). Dropping the intercept and the main effect
Table 1. Regression coefficients and $95 \%$ credible intervals for the relationship of plasma IgA activity with mucosal IgA activity, and the interaction of mucosal IgA activity with worm mass

\begin{tabular}{lc}
\hline \hline Regression term & Mean $(95 \%$ Credible interval) \\
\hline $\operatorname{IgA}_{\mathrm{m}}$ & $3.97(2 \cdot 91 ; 5 \cdot 03)$ \\
$\operatorname{IgA}_{\mathrm{m}} \times \log _{10}(\mathrm{WM})$ & $-1 \cdot 02(-1 \cdot 37 ;-0 \cdot 67)$ \\
\hline \hline
\end{tabular}

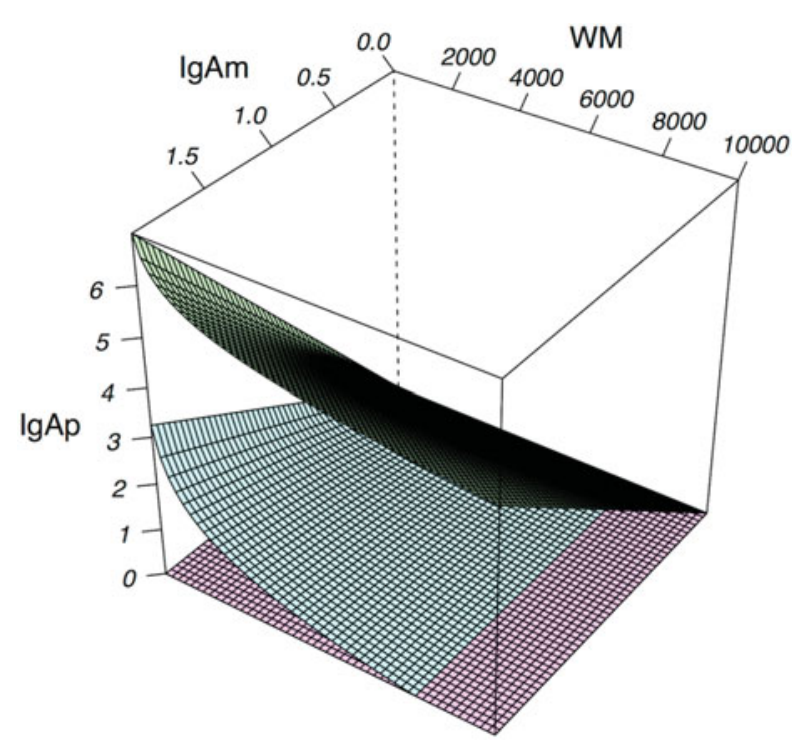

Fig. 2. Three-dimensional surface plot of the relationship between plasma $\operatorname{IgA}(\operatorname{IgAp})$, mucosal $\operatorname{IgA}$ (IgAm) and worm mass (WM - number of adult female $T$. circumcincta multiplied by worm length in $\mathrm{cm}$ ). The aquamarine (middle) surface shows the most probable values, the purple and green (lower and upper) surfaces were plotted with the lower and higher values in the $95 \%$ credible interval (Table 1) respectively.

of mucosal IgA reduced the DIC from $42 \cdot 8$ to $36 \cdot 6$ indicating that the full model is only 0.045 times as probable as the final model. The coefficient of determination $\left(R^{2}\right)$ for the final model is 0.83 : an $R^{2}$ of 0.83 implies a correlation of $0 \cdot 91$. This high correlation indicates that the major determinants of IgA activity in the plasma are in fact our two explanatory variables, IgA activity in the abomasal mucus and the mass of worms in the abomasum.

For a given worm mass, increased mucosal IgA was associated with increased IgA activity in the plasma (Fig. 2). Similarly, for a given concentration of mucosal IgA, increased worm mass was associated with decreased IgA activity in the plasma. Therefore low plasma IgA activity could be a consequence of low abomasal IgA activity or a high number of worms in the abomasum. Figure 2 demonstrates that there is very little IgA activity in the plasma when there is a high mass of nematodes in the abomasum.

The distribution of mucosal IgA activity in the abomasal mucus was predicted using the transfer equation based on values of plasma IgA activity, 


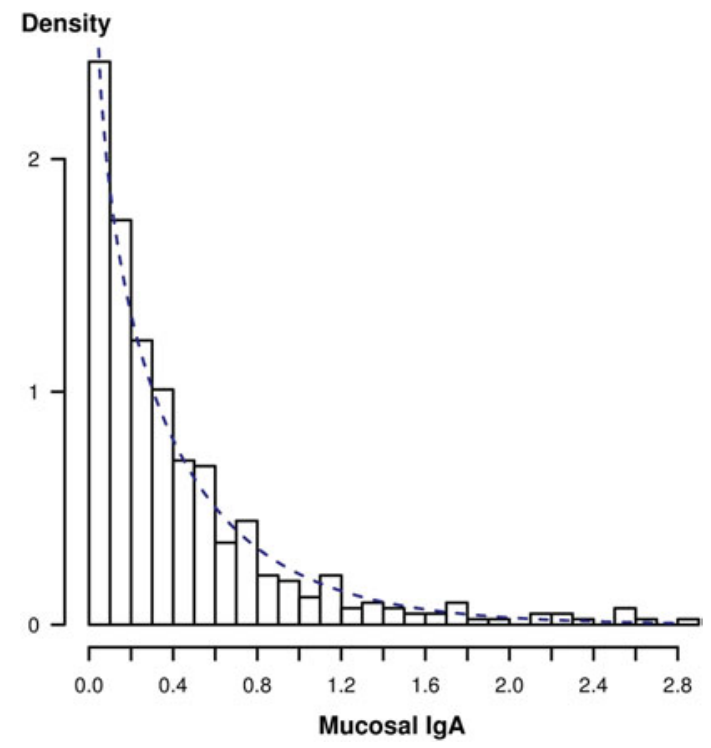

Fig. 3. Distribution of mucosal IgA from 0 to 3 OD (optical density). $88 \%$ of the data values are in this interval (negatives and extreme positives were dropped). Dashed line shows the fitted gamma distribution ( shape $=0.78$; rate $=1 \cdot 83)$. The Kolmogorov-Smirnov test $P$-value for the gamma fit was $0 \cdot 543$. The absence of extremely high values of mucosal IgA shortens the tail of the gamma distribution.

worm numbers and worm lengths from 484 naturally infected lambs from a single farm in Southwest Strathclyde. The predicted distribution of mucosal IgA did not follow a normal distribution (ShapiroWilk normality test $P<2 \cdot 2 \mathrm{e}-16)$, but was consistent with a gamma distribution (Kolmogorov-Smirnov test $P=0 \cdot 543$, Fig. 3).

\section{DISCUSSION}

This study has improved our understanding of the relationship between plasma and mucosal IgA following $T$. circumcincta infection in sheep, confirming that there is a strong, but non-linear, relationship, and has suggested that the distribution of mucosal IgA among naturally infected individuals is highly skewed.

The relationship between nematodes and the antibody response is complex. A strong IgA response against fourth-stage larvae will delay nematode development (Stear et al. 1995) and nematodes will spend longer as fourth-stage larvae and provide a greater antigenic stimulus. Conversely, a strong $\mathrm{IgE}$ response will prevent the establishment of third stage larvae and reduce the antigenic stimulus provided by fourth-stage larvae (Stear et al. 1995). In this experiment, sheep received challenges of 50000 larvae. In addition to antibody responses affecting the intensity of the antigenic challenge, there will be crossreactions between different species of nematodes (Stear et al. 2011) and possibly between different stages of the same parasite. For example, the IgA response against third stage larvae peaked 8-10 days after infection then persisted at a low level (Henderson and Stear, 2006). Most third-stage larvae will mature to fourth-stage larvae or be rejected in 4 days (Armour et al. 1966). IgA has a short half-life in plasma (Curtis and Bourne, 1973). Consequently the persistence of antibody to L3 may reflect crossreactions with molecules from other parasitic stages. In natural infections, there will be continuous challenge with incoming and developing larvae and most of the variation in $\operatorname{IgA}$ activity reflects genetic differences among lambs (Strain et al. 2002) rather than variation in exposure to infection.

An understanding of the relationship between plasma and mucosal IgA is necessary for drawing appropriate conclusions from measurements of plasma IgA. Previous research (Beh et al. 1974) has shown that in sheep, unlike humans (Snoeck et al. 2006), plasma IgA is derived from mucosal surfaces. The simplest explanation for the observed relationship between plasma IgA and mucosal IgA in our study is that abomasal IgA binds to nematodes and excretory-secretory molecules released from nematodes. The unbound IgA then equilibrates between mucus and interstitial fluid, and is then transferred to blood via the lymphatic system. This equilibration is helped by breaches that occur in the epithelial barrier during nematode infection (Stear et al. 2003); these breaches are caused by mast cell degranulation that break the junctions between the epithelial cells to facilitate the flow of effector cells. This also causes protein loss, which is one of the signs of nematode infections (Stear et al. 2003).

From an evolutionary perspective, sheep should produce enough IgA to counter an infection but should not invest excess resources in the immune system. Production of excessive IgA would produce large amounts of plasma IgA and a close relationship between mucosal and plasma IgA. The observation that very little IgA appears in the plasma during heavy infection suggests that most IgA is bound to nematodes and a relatively small proportion of nematode-specific IgA is transferred into plasma.

After applying the inverse transfer equation to naturally infected sheep, we obtained mucosal IgA values that were consistent with a gamma distribution. The observed gamma distribution implies that a few animals will have high mucosal IgA immune responses while the majority will be low responders. Low plasma IgA values occur in animals with a low intensity infection that triggers a small immune response or a heavily infected animal in which most of the mucosal IgA is bound to parasite antigens. High plasma IgA means that the response to infection is strong. A selection scheme that uses high responders for breeding would be potentially very successful in improving the overall response of the flock since the heritability of $\operatorname{IgA}$ responses is 
high (Strain et al. 2002). This is consistent with results obtained independently by Shaw et al. (2013), where they propose the use of salivary IgA to the nematode molecule CarLA as a measure of immunity to gastrointestinal nematodes. Salivary, plasma and mucosal IgA are correlated, with plasma having the higher correlation with mucosal IgA (MartinezValladares et al. 2005).

One area of concern is whether this type of selection will select for animals investing too many resources on the immune response. However, selection criteria would include production traits such as growth and the family history. The performance of the animals selected should therefore not decrease.

The transfer equation should provide a valuable component of mathematical models that simulate the behaviour of the immune system in small grazing animals facing infection, e.g. Singleton et al. (2011). One advantage of the nematode system is that it is possible to quantify variation among hosts in the number and size of the antigenic challenge. With the appropriate data, a similar study could be conducted in other grazing species and with other nematodes.

In conclusion, we have quantified the transfer of IgA from the abomasal mucus to the plasma. The amount of $\operatorname{IgA}$ in the plasma is strongly but nonlinearly related to the amount of $\operatorname{IgA}$ in the mucus and the interaction between mucus IgA and the parasite size and burden. The nature of this relationship has important implications for the design and outcome of diagnostic and control scenarios.

\section{FINANCIAL SUPPORT}

This work is supported by the EU funded Marie Curie Initial Training Networks (MC-ITN) programme and by the Wellcome Trust (WT091717MA).

\section{REFERENCES}

Armour, J., Jarrett, W. F. H. and Jennings, F. W. (1966). Experimental Ostertagia circumcincta infections in sheep: development and pathogenesis of a single infection. American fournal of Veterinary Research 27, 1267-1278. Balic, A., Bowles, V. M., Liu, Y. S. and Meeusen, E. N. T. (2003). Local immune responses in sensitized sheep following challenge infection with Teladorsagia circumcincta. Parasite Immunology 25, 375-381.

Beh, K. J., Watson, D. L. and Lascelles, A. (1974). Concentrations of immunoglobulins and albumin in lymph collected from various regions of the body of the sheep. Australian Fournal of Experimental Biology and Medical Science 52, 81-86.
Curtis, J. and Bourne, F. J. (1973). Half-lives of immunoglobulins IgG, IgA and IgM in the serum of new-born pigs. Immunology 24, 147-155.

Faraway, J. J. (2006). Extending the Linear Model with R. Chapman \& Hall/ CRC, Boca Raton, FL, USA.

Gelman, A., Carling, J. B., Stern, H.S. and Rubin, D. B. (2004). Bayesian Data Analysis. Chapman \& Hall/CRC, Boca Raton, FL, USA.

Henderson, N. G. and Stear, M. J. (2006). Eosinophil and IgA responses in sheep infected with Teladorsagia circumcincta. Veterinary Immunology and Immunopathology 112, 62-66.

Martinez-Valladares, M., Vara-Del Rio, M. P., Cruz-Rojo, M. A. and Rojo-Vazquez, F. A. (2005). Genetic resistance to Teladorsagia circumcincta: IgA and parameters at slaughter in Churra sheep. Parasite Immunology 27, 213-218. doi: 10.1111/j.1365-3024.2005.00769.x.

Murphy, L., Eckersall, P. D., Bishop, S. C., Pettit, J. J., Huntley, J., Burchmore, R. and Stear, M. J. (2010). Genetic variation among lambs in peripheral IgE activity against the larval stages of Teladorsagia circumcincta. Parasitology 137, 1249-1260.

Plummer, M. (2003). JAGS: a program for analysis of Bayesian graphical models using Gibbs sampling. Proceedings of the 3rd International Workshop on Distributed Statatistical Computing, DSC 2003.

R Development Core Team (2011). R: A Language and Environment for Statistical Computing. R Foundation for Statistical Computing, Vienna, Austria.

Shaw, R. J., Morris, C. A. and Wheeler, M. (2013). Genetic and phenotypic relationships between carbohydrate larval antigen (CarLA) IgA, parasite resistance and productivity in serial samples taken from lambs after weaning. International Fournal of Parasitology 43, 661-667. doi: 10.1016/j.ijpara.2013.03.003.

Singleton, D. R., Stear, M. J. and Matthews, L. (2011). A mechanistic model of developing immunity to Teladorsagia circumcincta infection in lambs. Parasitology 138, 322-332.

Sinski, E., Bairden, K., Duncan, J. L., Eisler, M. C., Holmes, P. H., McKellar, Q. A., Murray, M. and Stear, M. J. (1995). Local and plasma antibody-responses to the parasitic larval stages of the abomasal nematode Ostertagia circumcincta. Veterinary Parasitology 59, 107-118.

Snoeck, V., Peters, I. R. and Cox, E. (2006). The IgA system: a comparison of structure and function in different species. Veterinary Research 37, 455-467. doi: 10.1051/vetres:2006010.

Spiegelhalter, D. J., Best, N. G., Carlin, B. R. and van der Linde, A. (2002). Bayesian measures of model complexity and fit. Fournal of the Royal Statistics Society Series B - Statistics Methodology 64, 583-616.

Stear, M. J., Bishop, S.C., Doligalska, M., Duncan, J. L., Holmes, P. H., Irvine, J., McCririe, L., McKellar, Q. A., Sinski, E. and Murray, M. (1995). Regulation of egg production, worm burden, worm length and worm fecundity by host responses in sheep infected with Ostertagia circumcincta. Parasite Immunology 17, 643-652.

Stear, M. J., Bairden, K., Bishop, S. C., Gettinby, G., McKellar, Q. A., Park, M., Strain, S. A. J. and Wallace, D.S. (1998). The processes influencing the distribution of parasitic nematodes among naturally infected lambs. Parasitology 117, 165-171.

Stear, M. J., Bishop, S. C., Henderson, N. G. and Scott, I. (2003). A key mechanism of pathogenesis in sheep infected with the nematode Teladorsagia circumcincta. Animal Health Research Reviews 4, 45-52.

Stear, M. J., Singleton, D. R. and Matthews, L. (2011). An evolutionary perspective on gastrointestinal nematodes of sheep. Fournal of Helminthology 85, 113-120.

Strain, S. A. J. (2001). Immunity to abomasal parasites in lambs. Ph.D. thesis, Department of Veterinary Pre-Clinical Studies, University of Glasgow, Glasgow.

Strain, S. A. J., Bishop, S. C., Henderson, N. G., Kerr, A., McKellar, Q. A., Mitchell, S. and Stear, M. J. (2002). The genetic control of IgA activity against Teladorsagia circumcincta and its association with parasite resistance in naturally infected sheep. Parasitology 124, $545-552$. 